\title{
A New Iterative Scheme for Solving the Equilibrium Problems, Variational Inequality Problems, and Fixed Point Problems in Hilbert Spaces
}

\author{
Rabian Wangkeeree ${ }^{1,2}$ and Pakkapon Preechasilp ${ }^{1}$ \\ ${ }^{1}$ Department of Mathematics, Faculty of Science, Naresuan University, Phitsanulok 65000, Thailand \\ ${ }^{2}$ Centre of Excellence in Mathematics, CHE, Si Ayutthaya Road, Bangkok 10400, Thailand
}

Correspondence should be addressed to Rabian Wangkeeree, rabianw@nu.ac.th

Received 13 February 2012; Accepted 31 March 2012

Academic Editor: Zhenyu Huang

Copyright (C) 2012 R. Wangkeeree and P. Preechasilp. This is an open access article distributed under the Creative Commons Attribution License, which permits unrestricted use, distribution, and reproduction in any medium, provided the original work is properly cited.

We introduce the new iterative methods for finding a common solution set of monotone, Lipschitztype continuous equilibrium problems and the set of fixed point of nonexpansive mappings which is a unique solution of some variational inequality. We prove the strong convergence theorems of such iterative scheme in a real Hilbert space. The main result extends various results existing in the current literature.

\section{Introduction}

Let $H$ be a real Hilbert space and $C$ a nonempty closed convex subset of $H$ with inner product $\langle\cdot, \cdot\rangle$. Recall that a mapping $S: C \rightarrow C$ is called nonexpansive if $\|S x-S y\| \leq\|x-y\|$ for all $x, y \in C$. The set of all fixed points of $S$ is denoted by $F(S)=\{x \in C: x=S x\}$. A mapping $g: C \rightarrow C$ is a contraction on $C$ if there is a constant $\alpha \in(0,1)$ such that $\|g(x)-g(y)\| \leq \alpha\|x-y\|$ for all $x, y \in C$. We use $\Pi_{C}$ to denote the collection of all contractions on $C$. Note that each $g \in \Pi_{C}$ has a fixed unique fixed point in $C$. A linear bounded operator $A$ is strongly positive if there is a constant $\bar{\gamma}>0$ with property $\langle A x, x\rangle \geq \bar{\gamma}\|x\|^{2}$ for all $x \in H$.

Iterative methods for nonexpansive mappings have recently been applied to solve convex minimization problems; see, for example, [1-4] and the references therein. Convex minimization problems have a great impact and influence in the development of almost all branches of pure and applied sciences. A typical problem is to minimize a quadratic function over the set of the fixed points of a nonexpansive mapping on a real Hilbert space:

$$
\theta(x)=\min _{x \in C} \frac{1}{2}\langle A x, x\rangle-\langle x, b\rangle,
$$


where $A$ is a linear bounded operator, $C$ is the fixed point set of a nonexpansive mapping $T$, and $b$ is a given point in $H$. Let $H$ be a real Hilbert space. Recall that a linear bounded operator $B$ is strongly positive if there is a constant $\bar{\gamma}>0$ with property

$$
\langle A x, x\rangle \geq \bar{\gamma}\|x\|^{2} \quad \forall x \in H .
$$

Recently, Marino and $\mathrm{Xu}$ [5] introduced the following general iterative scheme based on the viscosity approximation method introduced by Moudafi [6]:

$$
x_{n+1}=\left(I-\alpha_{n} A\right) T x_{n}+\alpha_{n} \gamma g\left(x_{n}\right), \quad n \geq 0,
$$

where $A$ is a strongly positive bounded linear operator on $H$. They proved that if the sequence $\left\{\alpha_{n}\right\}$ of parameters satisfies appropriate conditions, then the sequence $\left\{x_{n}\right\}$ generated by (1.3) converges strongly to the unique solution of the variational inequality:

$$
\left\langle(A-\gamma g) x^{*}, x-x^{*}\right\rangle \geq 0, \quad x \in C,
$$

which is the optimality condition for the minimization problem:

$$
\min _{x \in C} \frac{1}{2}\langle A x, x\rangle-h(x)
$$

where $h$ is a potential function for $\gamma g$ (i.e., $h^{\prime}(x)=\gamma g(x)$ for $\left.x \in H\right)$.

A mapping $B$ of $C$ into $H$ is called monotone if $\langle B x-B y, x-y\rangle \geq 0$ for all $x, y \in C$. The variational inequality problem is to find $\tilde{x} \in C$ such that

$$
\langle B \tilde{x}, x-\tilde{x}\rangle \geq 0 \quad \forall x \in C .
$$

The set of solutions of variational inequality is denoted by $\operatorname{VI}(C, B)$. A mapping $B: C \rightarrow H$ is called inverse-strongly monotone if there exists a positive real number $\beta$ such that

$$
\langle x-y, B x-B y\rangle \geq \beta\|B x-B y\|^{2}, \quad \forall x, y \in C .
$$

For such a case, $B$ is $\beta$-inverse-strongly monotone. If $B$ is a $\beta$-inverse-strongly monotone mapping of $C$ to $H$, then it is obvious that $B$ is $(1 / \beta)$-Lipschitz continuous. In 2009, Klineam and Suantai [7] introduced the following general iterative method:

$$
x_{0} \in C, \quad x_{n+1}=P_{C}\left(\alpha_{n} \gamma g\left(x_{n}\right)+\left(I-\alpha_{n} A\right) S P_{C}\left(x_{n}-\lambda_{n} B x_{n}\right)\right), \quad n \geq 0,
$$

where $P_{C}$ is the metric projection of $H$ onto $C, g$ is a contraction, $A$ is a strongly positive linear bounded operator, $B$ is a $\beta$-inverse strongly monotone mapping, $\left\{\alpha_{n}\right\} \subset(0,1)$, and $\left\{\lambda_{n}\right\} \subset[a, b]$ for some $a, b$ with $0<a<b<2 \beta$. They proved that under certain appropriate conditions imposed on $\left\{\alpha_{n}\right\}$ and $\left\{\lambda_{n}\right\}$, the sequence generated by (1.8) converges strongly to a common element of the set of fixed points of nonexpansive mapping and the set of solutions 
of the variational inequality for an inverse strongly monotone mapping (say $\tilde{x} \in C$ ) which solves the following variational inequality:

$$
\langle(A-\gamma g) \tilde{x}, x-\tilde{x}\rangle \geq 0, \quad \forall x \in F(S) \cap \operatorname{VI}(C, B) .
$$

We recall the following well-known definitions. A bifunction $f: C \times C \rightarrow \mathbb{R}$ is called (a) monotone on $C$ if

$$
f(x, y)+f(y, x) \leq 0, \quad \forall x, y \in C
$$

(b) pseudomonotone on $C$ if

$$
f(x, y) \geq 0 \Longrightarrow f(y, x) \leq 0, \quad \forall x, y \in C
$$

(c) Lipschitz-type continuous on $C$ with two constants $c_{1}>0$ and $c_{2}>0$ if

$$
f(x, y)+f(y, z) \geq f(x, z)-c_{1}\|x-y\|^{2}-c_{2}\|y-z\|^{2}, \quad \forall x, y, z \in C
$$

We consider the following equilibrium problems: find $\tilde{x} \in C$ such that

$$
\text { Find } \tilde{x} \in C \text { such that } f(\tilde{x}, y) \geq 0, \quad \forall x \in C \text {. }
$$

The set of solution of problem (1.13) is denoted by $\operatorname{EP}(f, C)$. If $f(x, y):=\langle F x, y-x\rangle$ for all $x, y \in C$, where $F$ is a mapping from $C$ to $H$, then problem $\operatorname{EP}(f, C)$ reduces to the variational inequalities (1.6). It is well known that problem $\mathrm{EP}(f, C)$ covers many important problems in optimization and nonlinear analysis as well as it has found many applications in economic, transportation, and engineering.

For solving the common element of the set of fixed points of a nonexpansive mapping and the solution set of equilibrium problems, S. Takahashi and W. Takahashi [8] introduced the following viscosity approximation method:

$$
x_{0} \in H \text {, }
$$

Find $y_{n} \in C$ such that $f\left(y_{n}, y\right)+\frac{1}{r_{n}}\left\langle y-y_{n}, y_{n}-x_{n}\right\rangle, \quad \forall x \in C$,

$$
x_{n+1}=\alpha_{n} g\left(x_{n}\right)+\left(1-\alpha_{n}\right) S y_{n}, \quad \forall n \geq 0 \text {, }
$$

where $\left\{\alpha_{n}\right\} \subset[0,1]$ and $\left\{r_{n}\right\} \subset(0, \infty)$. They showed that under certain conditions over $\left\{\alpha_{n}\right\}$ and $\left\{r_{n}\right\}$, sequences $\left\{x_{n}\right\}$ and $\left\{y_{n}\right\}$ converge strongly to $z \in F(S) \cap \operatorname{EP}(F)$, where $z=P_{F(S) \cap E P(F)} g(z)$. 
In this paper, inspired and motivated by Klin-eam and Suantai [7] and S. Takahashi and W. Takahashi [8], we introduce the new algorithm for solving the common element of the set of fixed points of a nonexpansive mapping, the solution set of equilibrium problems, and the solution set of the variational inequality problems for an inverse strongly monotone mapping. Let $f$ be monotone, Lipschitz-type continuous on $C$ with two constants $c_{1}>0$ and $c_{2}>0, A$ a strongly linear bounded operator, and $B$ a $\beta$-inverse strongly monotone mapping. Let $g: C \rightarrow C$ be a contraction with coefficient $\alpha$ such that $0<\gamma<\bar{\gamma} / \alpha$ and $S: C \rightarrow C$ a nonexpansive mapping. The algorithm is now described as follows.

Step 1 (initialization). Choose positive sequences $\left\{\alpha_{n}\right\} \subset(0,1)$ and $\left\{\lambda_{n}\right\} \subset[c, d]$ for some $c, d \in(0,1 / L)$, where $L=\max \left\{2 c_{1}, 2 c_{2}\right\}$ and $\bar{\beta} \subset[a, b]$ for some $a, b$ with $0<a<b<2 \beta$.

Step 2 (solving convex problems). For a given point $x_{0}=x \in C$ and set $n:=0$, we solve the following two strongly convex problems:

$$
\begin{aligned}
& y_{n}=\operatorname{argmin}\left\{\lambda_{n} f\left(x_{n}, y\right)+\frac{1}{2}\left\|y-x_{n}\right\|^{2}: y \in C\right\}, \\
& t_{n}=\operatorname{argmin}\left\{\lambda_{n} f\left(y_{n}, y\right)+\frac{1}{2}\left\|y-x_{n}\right\|^{2}: y \in C\right\} .
\end{aligned}
$$

Step 3 (iteration $n$ ). Compute

$$
x_{n+1}=P_{C}\left(\alpha_{n} \gamma g\left(x_{n}\right)+\left(I-\alpha_{n} A\right) S P_{C}\left(t_{n}-\bar{\beta} B t_{n}\right)\right),
$$

where $P_{C}$ is the metric projection of $H$ onto $C$. Increase $n$ by 1 and go to Step 1 .

We show that under some control conditions the sequences $\left\{x_{n}\right\},\left\{y_{n}\right\}$, and $\left\{t_{n}\right\}$ defined by (1.15) and (1.16) converge strongly to a common element of solution set of monotone, Lipschitz-type continuous equilibrium problems, and the set of fixed points of nonexpansive mappings which is a unique solution of the variational inequality problem (1.6).

\section{Preliminaries}

Let $C$ be a nonempty closed convex subset of a real Hilbert space $H$. Let $f: C \times C \rightarrow \mathbb{R}$ be a bifunction. For solving the mixed equilibrium problem, let us assume the following conditions for a bifunction $f: C \times C \rightarrow \mathbb{R}$ :

(A1) $f(x, x)=0$ for all $x \in C$;

(A2) $f$ is Lipschitz-type continuous on $C$;

(A3) $f$ is monotone on $C$;

(A4) for each $x \in C, f(x, \cdot)$ is convex and subdifferentiable on $C$;

(A5) $f$ is upper semicontinuous on $C$.

The metric (nearest point) projection $P_{C}$ from a Hilbert space $H$ to a closed convex subset $C$ of $H$ is defined as follows: given $x \in H, P_{C} x$ is the only point in $C$ such that 
$\left\|x-P_{C} x\right\|-\inf \{\|x-y\|: y \in C\}$. In what follows lemma can be found in any standard functional analysis book.

Lemma 2.1. Let $C$ be a closed convex subset of a real Hilbert space $H$. Given $x \in H$ and $y \in C$, then

(i) $y=P_{C} x$ if and only if $\langle x-y, y-z\rangle \geq 0$ for all $z \in C$,

(ii) $P_{C}$ is nonexpansive,

(iii) $\left\langle x-y, P_{C} x-P_{C} y\right\rangle \geq\left\|P_{C} x-P_{C} y\right\|^{2}$ for all $x, y \in H$,

(iv) $\left\langle x-P_{C} x, P_{C} x-y\right\rangle$ for all $x \in H$ and $y \in C$.

Using Lemma 2.1, one can show that the variational inequality (1.6) is equivalent to a fixed point problem.

Lemma 2.2. The point $u \in C$ is a solution of the variational inequality (1.6) if and only if $u$ satisfies the relation $u=P_{C}(u-\lambda B u)$ for all $\lambda>0$.

A set-valued mapping $T: H \rightarrow 2^{H}$ is called monotone if for all $x, y \in H, u \in T x$, and $v \in T y$ imply $\langle x-y, u-v\rangle \geq 0$. A monotone mapping $T: H \rightarrow 2^{H}$ is maximal if the graph $G(T)$ of $T$ is not property contained in the graph of any other monotone mapping. It is known that a monotone mapping $T$ is maximal if and only if for $(x, u) \in H \times H,\langle x-y, u-v\rangle \geq 0$ for every $(y, v) \in G(T)$ implies $u \in T x$. Let $B$ be an inverse-strongly monotone mapping of $C$ to $H$, let $N_{C} v$ be normal cone to $C$ at $v \in C$, that is, $N_{C} v=\{w \in H:\langle v-u, w\rangle \geq 0$, for all $u \in C\}$, and define

$$
T v= \begin{cases}B v+N_{C} v, & \text { if } v \in C, \\ \emptyset, & \text { if } v \notin C .\end{cases}
$$

Then $T$ is a maximal monotone and $0 \in T v$ if and only if $v \in \mathrm{VI}(C, B)$ [9].

Now we collect some useful lemmas for proving the convergence results of this paper.

Lemma 2.3 (see [10]). Let $C$ be a nonempty closed convex subset of a real Hilbert space $H$ and $h: C \rightarrow \mathbb{R}$ be convex and subdifferentiable on $C$. Then $x^{*}$ is a solution to the following convex problem:

$$
\min \{h(x): x \in C\}
$$

if and only if $0 \in \partial h\left(x^{*}\right)+N_{C}\left(x^{*}\right)$, where $\partial h(\cdot)$ denotes the subdifferential of $h$ and $N_{C}\left(x^{*}\right)$ is the (outward) normal cone of $C$ at $x^{*} \in C$.

Lemma 2.4 (see [11, Lemma 3.1]). Let $C$ be a nonempty closed convex subset of a real Hilbert space $H$. Let $f: C \times C \rightarrow \mathbb{R}$ be a pseudomonotone, Lipschitz-type continuous bifunction with constants $c_{1}>0$ and $c_{2}>0$. For each $x \in C$, let $f(x, \cdot)$ be convex and subdifferentiable on $C$. Suppose that the sequences $\left\{x_{n}\right\},\left\{y_{n}\right\}$, and $\left\{t_{n}\right\}$ are generated by Scheme (1.15) and $p \in \operatorname{EP}(f)$. Then

$$
\left\|t_{n}-p\right\|^{2} \leq\left\|x_{n}-p\right\|^{2}-\left(1-2 \lambda_{n} c_{1}\right)\left\|x_{n}-y_{n}\right\|^{2}-\left(1-2 \lambda_{n} c_{2}\right)\left\|y_{n}-t_{n}\right\|^{2}, \quad \forall n \geq 0 .
$$


Lemma 2.5 (see [12]). Let $C$ be a closed convex subset of a Hilbert space $H$ and let $S: C \rightarrow C$ be a nonexpansive mapping such that $F(S) \neq \emptyset$. If a sequence $\left\{x_{n}\right\}$ in $C$ such that $x_{n} \rightarrow z$ and $x_{n}-S x_{n} \rightarrow$ 0 , then $z=S z$.

Lemma 2.6 (see [5]). Assume that $A$ is a strongly positive linear bounded operator on a Hilbert space $H$ with coefficient $\bar{\gamma}>0$ and $0<\rho \leq\|A\|^{-1}$, then $\|I-\rho A\| \leq 1-\rho \bar{\gamma}$.

In the following, we also need the following lemma that can be found in the existing literature $[3,13]$.

Lemma 2.7 (see [3, Lemma 2.1]). Let $\left\{a_{n}\right\}$ be a sequence of non-negative real number satisfying the following property:

$$
a_{n+1} \leq\left(1-\gamma_{n}\right) a_{n}+\gamma_{n} \beta_{n}, \quad n \geq 0,
$$

where $\left\{\gamma_{n}\right\} \subseteq(0,1)$ and $\left\{\beta_{n}\right\} \subseteq \mathbb{R}$ such that $\sum_{n=0}^{\infty} \gamma_{n}=\infty$ and $\lim \sup _{n \rightarrow \infty} \beta_{n} \leq 0$. Then $\left\{a_{n}\right\}$ converges to zero, as $n \rightarrow \infty$.

\section{Main Theorems}

In this section, we prove the strong convergence theorem for solving a common element of solution set of monotone, Lipschitz-type continuous equilibrium problems and the set of fixed points of nonexpansive mappings.

Theorem 3.1. Let $H$ be a real Hilbert space, and let $C$ be a closed convex subset of $H$. Let $f: C \times C \rightarrow$ $\mathbb{R}$ be a bifunction satisfying (A1)-(A5), let $B: C \rightarrow H$ be a $\beta$-inverse strongly monotone mapping, let $A$ be a strongly positive linear bounded operator of $H$ into itself with coefficient $\bar{\gamma}>0$ such that $\|A\|=1$ and let $g: C \rightarrow C$ be a contraction with coefficient $\alpha(\alpha \in(0,1))$. Assume that $0<\gamma<\bar{\gamma} / \alpha$. Let $S$ be a nonexpansive mapping of $C$ into itself such that $\Omega:=F(S) \cap \operatorname{EP}(f) \cap \operatorname{VI}(C, B) \neq \emptyset$. Let the sequences $\left\{x_{n}\right\},\left\{y_{n}\right\}$, and $\left\{t_{n}\right\}$ be generated by (1.15) and (1.16), where $\left\{\alpha_{n}\right\} \subset(0,1),\{\bar{\beta}\} \subset[a, b]$ for some $a, b \in(0,2 \beta)$, and $\left\{\lambda_{n}\right\} \subset[c, d]$ for some $c, d \in(0,1 / L)$, where $L=\max \left\{2 c_{1}, 2 c_{2}\right\}$. Suppose that the following conditions are satisfied:

(B1) $\lim _{n \rightarrow \infty} \alpha_{n}=0$;

(B2) $\sum_{n=1}^{\infty} \alpha_{n}=\infty$;

(B3) $\sum_{n=1}^{\infty}\left|\alpha_{n+1}-\alpha_{n}\right|<\infty$;

(B4) $\sum_{n=1}^{\infty} \sqrt{\left|\lambda_{n+1}-\lambda_{n}\right|}<\infty$.

Then the following holds.

(i) $P_{\Omega}(I-A+\gamma g)$ is a contraction on $C$; hence there exists $q \in C$ such that $q=P_{\Omega}(I-A+$ $r g)(q)$, where $P_{\Omega}$ is the metric projection of $H$ onto $C$.

(ii) The sequences $\left\{x_{n}\right\},\left\{y_{n}\right\}$, and $\left\{t_{n}\right\}$ converge strongly to the same point $q$. 
Journal of Applied Mathematics

Proof. For any $x, y \in H$, we have

$$
\begin{aligned}
\left\|P_{\Omega}(\gamma g+(I-A)) x-P_{\Omega}(\gamma g+(I-A)) y\right\| & \leq\|\gamma g+(I-A) x-(\gamma g+(I-A)) y\| \\
& \leq \gamma\|g(x)-g(y)\|+\|I-A\|\|x-y\| \\
& \leq \gamma \alpha\|x-y\|+(1-\bar{\gamma})\|x-y\| \\
& =(1-(\bar{\gamma}-\gamma \alpha))\|x-y\| .
\end{aligned}
$$

Banach's contraction principle guarantees that $P_{\Omega}(\gamma g+(I-A))$ has a unique fixed point, say $q \in H$. That is, $q=P_{\Omega}(\gamma g+(I-A))(q)$. By Lemma 2.1(i), we obtain that

$$
\langle(\gamma g-A) q, p-q\rangle \leq 0, \quad \forall p \in \Omega
$$

The proof of (ii) is divided into several steps.

Step 1. $I-\bar{\beta} B$ is nonexpansive mapping. Indeed, since $B$ is a $\beta$-strongly monotone mapping and $0<\bar{\beta}<2 \beta$, for all $x, y \in C$, we have

$$
\begin{aligned}
\|(I-\bar{\beta} B) x-(I-\bar{\beta} B) y\|^{2} & =\|(x-y)-\bar{\beta}(B x-B y)\|^{2} \\
& =\|x-y\|^{2}-2 \bar{\beta}\langle x-y, B x-B y\rangle+\bar{\beta}^{2}\|B x-B y\|^{2} \\
& \leq\|x-y\|^{2}-2 \bar{\beta}\|B x-B y\|^{2}+\bar{\beta}^{2}\|B x-B y\|^{2} \\
& \leq\|x-y\|^{2}+\bar{\beta}(\bar{\beta}-2 \beta)\|B x-B y\|^{2} \\
& \leq\|x-y\|^{2} .
\end{aligned}
$$

Step 2. We show that $\left\{x_{n}\right\}$ is a bounded sequence. Put $w_{n}=P_{C}\left(t_{n}-\bar{\beta} B t_{n}\right)$ for all $n \geq 0$. Let $p \in \Omega$; we have

$$
\begin{aligned}
\left\|w_{n}-p\right\| & =\left\|P_{C}\left(t_{n}-\bar{\beta} B t_{n}\right)-P_{C}(p-\bar{\beta} B p)\right\| \\
& \leq\left\|(I-\bar{\beta} B) t_{n}-(I-\bar{\beta} B) p\right\| \\
& \leq\left\|t_{n}-p\right\| .
\end{aligned}
$$

By Lemma 2.4, we have

$$
\begin{aligned}
\left\|x_{n+1}-p\right\| & =\left\|P_{C}\left(\alpha_{n} \gamma g\left(x_{n}\right)+\left(I-\alpha_{n} A\right) S\left(w_{n}\right)\right)-p\right\| \\
& \leq\left\|\alpha_{n} \gamma g\left(x_{n}\right)+\left(I-\alpha_{n} A\right) S\left(w_{n}\right)-p\right\| \\
& \leq \alpha_{n}\left\|\gamma g\left(x_{n}\right)-A p\right\|+\left\|I-\alpha_{n} A\right\|\left\|S\left(w_{n}\right)-S(p)\right\|
\end{aligned}
$$




$$
\begin{aligned}
& \leq \alpha_{n}\left\|\gamma g\left(x_{n}\right)-A p\right\|+\left\|I-\alpha_{n} A\right\|\left\|w_{n}-p\right\| \\
& \leq \alpha_{n}\left\|\gamma g\left(x_{n}\right)-A p\right\|+\left(1-\alpha_{n} \bar{\gamma}\right)\left\|t_{n}-p\right\| \\
& \leq \alpha_{n}\left\|\gamma g\left(x_{n}\right)-A p\right\|+\left(1-\alpha_{n} \bar{\gamma}\right)\left\|x_{n}-p\right\| \\
& \leq \alpha_{n} \gamma \alpha\left\|x_{n}-p\right\|+\alpha_{n}\|\gamma g(p)-A p\|+\left(1-\alpha_{n} \bar{\gamma}\right)\left\|x_{n}-p\right\| \\
& \leq\left(1-\alpha_{n}(\bar{\gamma}-\gamma \alpha)\right)\left\|x_{n}-p\right\|+\alpha_{n}(\bar{\gamma}-\gamma \alpha) \frac{\|\gamma g(p)-A p\|}{\bar{\gamma}-\gamma \alpha} .
\end{aligned}
$$

By induction, we get that

$$
\left\|x_{n}-p\right\| \leq \max \left\{\left\|x_{1}-p\right\|, \frac{1}{\bar{\gamma}-\gamma \alpha}\|\gamma g(p)-A p\|\right\}, \quad n \geq 0 .
$$

Hence $\left\{x_{n}\right\}$ is bounded, and then $\left\{w_{n}\right\},\left\{y_{n}\right\}$, and $\left\{t_{n}\right\}$ are also bounded.

Step 3. We show that

$$
\lim _{n \rightarrow \infty}\left\|x_{n+1}-x_{n}\right\|=0
$$

Since $f(x, \cdot)$ is convex on $C$ for each $x \in C$, applying Lemma 2.3, we see that $t_{n}=$ $\operatorname{argmin}\left\{(1 / 2)\left\|t-x_{n}\right\|+\lambda_{n} f\left(y_{n}, t\right): t \in C\right\}$ if and only if

$$
0 \in \partial_{2}\left(\lambda_{n} f\left(y_{n}, t\right)+\frac{1}{2}\left\|y-x_{n}\right\|^{2}\right)\left(t_{n}\right)+N_{C}\left(t_{n}\right)
$$

where $N_{C}(x)$ is the (outward) normal cone of $C$ at $x \in C$. This implies that $0=\lambda_{n} w+t_{n}-x_{n}+\bar{w}$, where $w \in \partial_{2} f\left(y_{n}, t_{n}\right)$ and $\bar{w} \in N_{C} t_{n}$. By the definition of the normal cone $N_{C}$, we have

$$
\left\langle x_{n}-\lambda_{n} w-t_{n}, t-t_{n}\right\rangle=\left\langle\bar{w}, t-t_{n}\right\rangle \leq 0, \quad \forall t \in C,
$$

and so

$$
\left\langle t_{n}-x_{n}, t-t_{n}\right\rangle \geq \lambda_{n}\left\langle w, t_{n}-t\right\rangle, \quad \forall t \in C
$$

Substituting $t=t_{n+1} \in C$ into (3.10), we get that

$$
\left\langle t_{n}-x_{n}, t_{n+1}-t_{n}\right\rangle \geq \lambda_{n}\left\langle w, t_{n}-t_{n+1}\right\rangle
$$

Since $f(x, \cdot)$ is subdifferentiable on $C$ and $w \in \partial_{2} f\left(y_{n}, t_{n}\right)$, we have

$$
f\left(y_{n}, t\right)-f\left(y_{n}, t_{n}\right) \geq\left\langle w, t-t_{n}\right\rangle, \quad \forall t \in C
$$


Journal of Applied Mathematics

From (3.11) and (3.12), we obtain that

$$
\begin{aligned}
\left\langle t_{n}-x_{n}, t_{n+1}-t_{n}\right\rangle & \geq \lambda_{n}\left\langle w, t_{n}-t_{n+1}\right\rangle \\
& \geq \lambda_{n}\left(f\left(y_{n}, t_{n}\right)-f\left(y_{n}, t_{n+1}\right)\right) .
\end{aligned}
$$

By the similar way, we also have

$$
\left\langle t_{n+1}-x_{n+1}, t_{n}-t_{n+1}\right\rangle \geq \lambda_{n+1}\left(f\left(y_{n+1}, t_{n+1}\right)-f\left(y_{n+1}, t_{n}\right)\right) .
$$

It follows from (3.13) and (3.14) and $f$ is Lipschitz-type continuous and monotone, we get

$$
\begin{aligned}
\frac{1}{2}\left\|x_{n+1}-x_{n}\right\|^{2}-\frac{1}{2}\left\|t_{n+1}-t_{n}\right\|^{2} \geq & \left\langle t_{n+1}-t_{n}, t_{n}-x_{n}-t_{n+1}+x_{n+1}\right\rangle \\
\geq & \lambda_{n}\left(f\left(y_{n}, t_{n}\right)-f\left(y_{n}, t_{n+1}\right)\right)+\lambda_{n+1}\left(f\left(y_{n+1}, t_{n+1}\right)-f\left(y_{n+1}, t_{n}\right)\right) \\
\geq & \lambda_{n}\left(-f\left(t_{n}, t_{n+1}\right)-c_{1}\left\|y_{n}-t_{n}\right\|^{2}-c_{2}\left\|t_{n}-t_{n+1}\right\|^{2}\right) \\
& +\lambda_{n+1}\left(-f\left(t_{n+1}, t_{n}\right)-c_{1}\left\|y_{n+1}, t_{n+1}\right\|^{2}-c_{2}\left\|t_{n}-t_{n+1}\right\|^{2}\right) \\
\geq & \left(\lambda_{n+1}-\lambda_{n}\right) f\left(t_{n}, t_{n+1}\right) \\
\geq & -\left|\lambda_{n+1}-\lambda_{n}\right|\left|f\left(t_{n}, t_{n+1}\right)\right|,
\end{aligned}
$$

and hence

$$
\begin{aligned}
\left\|t_{n+1}-t_{n}\right\|^{2} & =\sqrt{\left\|x_{n+1}-x_{n}\right\|^{2}+2\left|\lambda_{n+1}-\lambda_{n}\right|\left|f\left(t_{n}, t_{n+1}\right)\right|} \\
& \leq\left\|x_{n+1}-x_{n}\right\| \sqrt{2\left|\lambda_{n+1}-\lambda_{n}\right|\left|f\left(t_{n}, t_{n+1}\right)\right|} .
\end{aligned}
$$

Thus, we have

$$
\begin{aligned}
\left\|x_{n+1}-x_{n}\right\| \\
=\left\|P_{C}\left(\alpha_{n} \gamma g\left(x_{n}\right)+\left(I-\alpha_{n} A\right) S\left(w_{n}\right)\right)-P_{C}\left(\alpha_{n-1} \gamma g\left(x_{n-1}\right)+\left(I-\alpha_{n-1} A\right) S\left(w_{n-1}\right)\right)\right\| \\
\leq\left\|\alpha_{n} \gamma g\left(x_{n}\right)+\left(I-\alpha_{n} A\right) S\left(w_{n}\right)-\alpha_{n-1} \gamma g\left(x_{n-1}\right)-\left(I-\alpha_{n-1} A\right) S\left(w_{n-1}\right)\right\| \\
=\| \alpha_{n} \gamma g\left(x_{n}\right)-\alpha_{n} \gamma g\left(x_{n-1}\right)+\alpha_{n} \gamma g\left(x_{n-1}\right)-\alpha_{n-1} \gamma g\left(x_{n-1}\right)+\left(I-\alpha_{n} A\right) S\left(t_{n}\right) \\
\quad \quad\left(I-\alpha_{n} A\right) S\left(t_{n-1}\right)+\left(I-\alpha_{n} A\right) S\left(t_{n-1}\right)-\left(I-\alpha_{n-1} A\right) S\left(t_{n-1}\right) \| \\
\leq \alpha_{n} \gamma \alpha\left\|x_{n}-x_{n-1}\right\|+\left|\alpha_{n}-\alpha_{n-1}\right|\left\|\gamma g\left(x_{n-1}\right)\right\| \\
\quad+\left\|I-\alpha_{n} A\right\|\left\|S\left(w_{n}\right)-S\left(w_{n-1}\right)\right\|+\left|\alpha_{n}-\alpha_{n-1}\right|\left\|A S\left(t_{n-1}\right)\right\| \\
\leq \alpha_{n} \gamma \alpha\left\|x_{n}-x_{n-1}\right\|+\left\|I-\alpha_{n} \bar{\gamma}\right\|\left\|w_{n}-w_{n-1}\right\|+\left|\alpha_{n}-\alpha_{n-1}\right|\left(\gamma\left\|g\left(x_{n-1}\right)\right\|+\left\|A S\left(t_{n-1}\right)\right\|\right)
\end{aligned}
$$




$$
\begin{aligned}
\leq & \alpha_{n} \gamma \alpha\left\|x_{n}-x_{n-1}\right\|+\left\|I-\alpha_{n} \bar{\gamma}\right\|\left\|t_{n}-t_{n-1}\right\|+\left|\alpha_{n}-\alpha_{n-1}\right|\left(\gamma\left\|g\left(x_{n-1}\right)\right\|+\left\|A S\left(t_{n-1}\right)\right\|\right) \\
\leq & \alpha_{n} \gamma \alpha\left\|x_{n}-x_{n-1}\right\|+\left(1-\alpha_{n} \bar{\gamma}\right)\left(\left\|x_{n}-x_{n-1}\right\|+\sqrt{2\left|\lambda_{n+1}-\lambda_{n}\right|\left|f\left(t_{n}, t_{n+1}\right)\right|}\right) \\
& +\left|\alpha_{n}-\alpha_{n-1}\right|\left(\gamma\left\|g\left(x_{n-1}\right)\right\|+\left\|A S\left(t_{n+1}\right)\right\|\right) \\
\leq & \left(1-\alpha_{n}(\bar{\gamma}-\gamma \alpha)\right)\left\|x_{n}-x_{n-1}\right\|+\left(1-\alpha_{n} \bar{\gamma}\right) \sqrt{2\left|\lambda_{n+1}-\lambda_{n}\right|} K+\left|\alpha_{n}-\alpha_{n-1}\right| M
\end{aligned}
$$

where $K \geq \sup _{n \geq 0} \sqrt{\left|f\left(t_{n-1}, t_{n}\right)\right|}$ and $M \geq \sup _{n \geq 0} \gamma\left\|g\left(x_{n-1}\right)\right\|+\left\|A S\left(t_{n-1}\right)\right\|$. Using (B3), (B4), and Lemma 2.7, we have $\lim _{n \rightarrow \infty}\left\|x_{n+1}-x_{n}\right\|=0$.

Step 4. We show that

$$
\lim _{n \rightarrow \infty}\left\|x_{n}-t_{n}\right\|=0
$$

Indeed, for each $p \in \Omega$, applying Lemma 2.4, we have

$$
\begin{aligned}
& \left\|x_{n+1}-p\right\|^{2} \\
& =\left\|P_{C}\left(\alpha_{n} \gamma g\left(x_{n}\right)+\left(I-\alpha_{n} A\right) S\left(w_{n}\right)\right)-p\right\|^{2} \\
& \leq\left\|\alpha_{n} \gamma g\left(x_{n}\right)+\left(I-\alpha_{n} A\right) S\left(w_{n}\right)-p\right\|^{2} \\
& \leq\left(\alpha_{n}\left\|\gamma g\left(x_{n}\right)-A p\right\|+\left(1-\alpha_{n} \bar{\gamma}\right)\left\|S\left(w_{n}\right)-p\right\|\right)^{2} \\
& =\alpha_{n}^{2}\left\|\gamma g\left(x_{n}\right)-A p\right\|^{2}+\left(1-\alpha_{n} \bar{\gamma}\right)^{2}\left\|S\left(w_{n}\right)-p\right\|^{2} \\
& +2 \alpha_{n}\left(1-\alpha_{n} \bar{\gamma}\right)\left\|\gamma g\left(x_{n}\right)-g(p)\right\|\left\|S\left(w_{n}\right)-p\right\| \\
& \leq \alpha_{n}\left\|\gamma g\left(x_{n}\right)-A p\right\|^{2}+\left(1-\alpha_{n} \bar{\gamma}\right)\left\|S\left(w_{n}\right)-p\right\|^{2} \\
& +2 \alpha_{n}\left(1-\alpha_{n} \bar{\gamma}\right)\left\|\gamma g\left(x_{n}\right)-g(p)\right\|\left\|S\left(w_{n}\right)-p\right\| \\
& \leq \alpha_{n}\left(\left\|\gamma g\left(x_{n}\right)-\gamma g(p)\right\|+\|\gamma g(p)-A p\|\right)^{2}+\left(1-\alpha_{n} \bar{\gamma}\right)\left\|S\left(w_{n}\right)-p\right\|^{2} \\
& +2 \alpha_{n}\left(1-\alpha_{n} \bar{\gamma}\right)\left\|\gamma g\left(x_{n}\right)-g(p)\right\|\left\|S\left(w_{n}\right)-p\right\| \\
& =\alpha_{n}\left\|\gamma g\left(x_{n}\right)-\gamma g(p)\right\|^{2}+2 \alpha_{n}\left\|\gamma g\left(x_{n}\right)-\gamma g(p)\right\|\|\gamma g(p)-A p\|+\alpha_{n}\|\gamma g(p)-A p\|^{2} \\
& +\left(1-\alpha_{n} \bar{\gamma}\right)\left\|S\left(w_{n}\right)-p\right\|^{2}+2 \alpha_{n}\left(1-\alpha_{n} \bar{\gamma}\right)\left\|\gamma g\left(x_{n}\right)-g(p)\right\|\left\|S\left(w_{n}\right)-p\right\| \\
& \leq \alpha_{n}\left\|\gamma g\left(x_{n}\right)-\gamma g(p)\right\|^{2}+\left(1-\alpha_{n} \bar{\gamma}\right)\left\|t_{n}-p\right\|^{2}+\theta_{n} \\
& \leq \alpha_{n} \gamma^{2} \alpha^{2}\left\|x_{n}-p\right\|^{2}+\theta_{n} \\
& +\left(1-\alpha_{n} \bar{\gamma}\right)\left(\left\|x_{n}-p\right\|^{2}-\left(1-2 \lambda_{n} c_{1}\right)\left\|x_{n}-y_{n}\right\|^{2}-\left(1-2 \lambda_{n} c_{2}\right)\left\|y_{n}-t_{n}\right\|^{2}\right)
\end{aligned}
$$


Journal of Applied Mathematics

$$
\begin{aligned}
\leq & \alpha_{n}(\gamma \alpha)^{2}\left\|x_{n}-p\right\|^{2}+\left(1-\alpha_{n} \bar{\gamma}\right)\left\|x_{n}-p\right\|^{2}-\left(1-\alpha_{n} \bar{\gamma}\right)\left(1-2 \lambda_{n} c_{1}\right)\left\|x_{n}-y_{n}\right\|^{2} \\
& -\left(1-\alpha_{n} \bar{\gamma}\right)\left(1-2 \lambda_{n} c_{2}\right)\left\|y_{n}-t_{n}\right\|^{2}+\theta_{n} \\
= & \left(1-\alpha_{n}\left(\bar{\gamma}-(\gamma \alpha)^{2}\right)\right)\left\|x_{n}-p\right\|^{2}-\left(1-\alpha_{n} \bar{\gamma}\right)\left(1-2 \lambda_{n} c_{1}\right)\left\|x_{n}-y_{n}\right\|^{2} \\
& -\left(1-\alpha_{n} \bar{\gamma}\right)\left(1-2 \lambda_{n} c_{2}\right)\left\|y_{n}-t_{n}\right\|^{2}+\theta_{n} \\
\leq & \left\|x_{n}-p\right\|^{2}-\left(1-\alpha_{n} \bar{\gamma}\right)\left(1-2 \lambda_{n} c_{1}\right)\left\|x_{n}-y_{n}\right\|^{2}-\left(1-\alpha_{n} \bar{\gamma}\right)\left(1-2 \lambda_{n} c_{2}\right)\left\|y_{n}-t_{n}\right\|^{2} \\
& +\theta_{n},
\end{aligned}
$$

where

$$
\begin{aligned}
\theta_{n}= & 2 \alpha_{n}\left\|\gamma g\left(x_{n}\right)-r g(p)\right\|\|\gamma g(p)-A p\|+2 \alpha_{n}\left(1-\alpha_{n} \bar{\gamma}\right)\left\|\gamma g\left(x_{n}\right)-g(p)\right\| \\
& \times\left\|S\left(w_{n}\right)-p\right\|+\alpha_{n}\|\gamma g(p)-A p\|^{2} .
\end{aligned}
$$

It then follows that

$$
\begin{aligned}
\left(1-\alpha_{n} \bar{\gamma}\right)\left(1-2 \lambda_{n} c_{2}\right)\left\|x_{n}-y_{n}\right\|^{2} \leq & \left\|x_{n}-p\right\|^{2}-\left\|x_{n+1}-p\right\|^{2} \\
& -\left(1-\alpha_{n} \bar{\gamma}\right)\left(1-2 \lambda_{n} c_{1}\right)\left\|y_{n}-t_{n}\right\|^{2}+\theta_{n} \\
\leq & \left(\left\|x_{n}-p\right\|+\left\|x_{n+1}-p\right\|\right)\left\|x_{n}-x_{n+1}\right\|+\theta_{n} \longrightarrow 0,
\end{aligned}
$$

as $n \rightarrow \infty$. Hence

$$
\lim _{n \rightarrow \infty}\left\|x_{n}-y_{n}\right\|=0
$$

By the similar way, also

$$
\lim _{n \rightarrow \infty}\left\|y_{n}-t_{n}\right\|=0
$$

From (3.22) and (3.23), we can conclude that

$$
\left\|x_{n}-t_{n}\right\| \leq\left\|x_{n}-y_{n}\right\|+\left\|y_{n}-t_{n}\right\| \longrightarrow 0 \quad \text { as } n \longrightarrow \infty .
$$

Step 5. We show that

$$
\lim _{n \rightarrow \infty}\left\|x_{n}-S x_{n}\right\|=0
$$

From (1.15), we get that

$$
\begin{aligned}
\left\|x_{n+1}-p\right\|^{2} & =\left\|P_{C}\left(\alpha_{n} \gamma g\left(x_{n}\right)+\left(I-\alpha_{n} A\right) S w_{n}\right)-p\right\|^{2} \\
& \leq\left\|\alpha_{n} \gamma g\left(x_{n}\right)+\left(I-\alpha_{n} A\right) S w_{n}-p\right\|^{2}
\end{aligned}
$$




$$
\begin{aligned}
\leq & \left(\alpha_{n}\left\|\gamma g\left(x_{n}\right)-A p\right\|+\left(1-\alpha_{n} \bar{\gamma}\right)\left\|S w_{n}-p\right\|\right)^{2} \\
\leq & \alpha_{n}\left\|\gamma g\left(x_{n}\right)-A p\right\|^{2}+\left(1-\alpha_{n} \bar{\gamma}\right)\left\|w_{n}-p\right\|^{2} \\
& +2 \alpha_{n}\left(1-\alpha_{n} \bar{\gamma}\right)\left\|\gamma g\left(x_{n}\right)-A p\right\|\left\|w_{n}-p\right\| \\
\leq & \alpha_{n}\left\|\gamma g\left(x_{n}\right)-A p\right\|^{2}+\left(1-\alpha_{n} \bar{\gamma}\right)\left\|(I-\bar{\beta} B) t_{n}-(I-\bar{\beta} B) p\right\|^{2} \\
& +2 \alpha_{n}\left(1-\alpha_{n} \bar{\gamma}\right)\left\|\gamma g\left(x_{n}\right)-A p\right\|\left\|w_{n}-p\right\| \\
\leq & \alpha_{n}\left\|\gamma g\left(x_{n}\right)-A p\right\|^{2}+\left(1-\alpha_{n} \bar{\gamma}\right)\left(\left\|t_{n}-p\right\|^{2}+\bar{\beta}(\bar{\beta}-2 \beta)\left\|B t_{n}-B p\right\|^{2}\right) \\
\leq & \alpha_{n}\left\|\gamma g\left(x_{n}\right)-A p\right\|^{2}+\left\|t_{n}-p\right\|^{2}+\left(1-\alpha_{n} \bar{\gamma}\right) a(b-2 \beta)\left\|B t_{n}-B p\right\|^{2} \\
& +2 \alpha_{n}\left(1-\alpha_{n} \bar{\gamma}\right)\left\|\gamma g\left(x_{n}\right)-A p\right\|\left\|w_{n}-p\right\| \\
\leq & \alpha_{n}\left\|\gamma g\left(x_{n}\right)-A p\right\|^{2}+\left\|x_{n}-p\right\|^{2}+\left(1-\alpha_{n} \bar{\gamma}\right) a(b-2 \beta)\left\|B t_{n}-B p\right\|^{2} \\
& +2 \alpha_{n}\left(1-\alpha_{n} \bar{\gamma}\right)\left\|\gamma g\left(x_{n}\right)-A p\right\|\left\|w_{n}-p\right\|,
\end{aligned}
$$

and hence

$$
\begin{aligned}
-\left(1-\alpha_{n} \bar{\gamma}\right) a(b-2 \beta)\left\|B t_{n}-B p\right\|^{2} \leq & \alpha_{n}\left\|\gamma g\left(x_{n}\right)-A p\right\|+\left\|x_{n}-p\right\|^{2}-\left\|x_{n+1}-p\right\|^{2} \\
& +2 \alpha_{n}\left(1-\alpha_{n} \bar{\gamma}\right)\left\|\gamma g\left(x_{n}\right)-A p\right\|\left\|w_{n}-p\right\| .
\end{aligned}
$$

Since $\alpha_{n} \rightarrow 0$, we get that $\left\|B t_{n}-B p\right\| \rightarrow 0$, as $n \rightarrow \infty$. By Lemma 2.1(iii), we have

$$
\begin{aligned}
& \left\|w_{n}-p\right\|^{2} \\
& \quad=\left\|P_{C}\left(t_{n}-\bar{\beta} B t_{n}\right)-P_{C}(p-\bar{\beta} B p)\right\|^{2} \\
& \quad \leq\left\langle\left(t_{n}-\bar{\beta} B t_{n}\right)-(p-\bar{\beta} B p), w_{n}-p\right\rangle \\
& \quad=\frac{1}{2}\left(\left\|\left(t_{n}-\bar{\beta} B t_{n}\right)-(p-\bar{\beta} B p)\right\|^{2}+\left\|w_{n}-p\right\|^{2}-\left\|\left(t_{n}-\bar{\beta} B t_{n}\right)-\left(t_{n}-\bar{\beta} B t_{n}\right)-\left(w_{n}-p\right)\right\|^{2}\right) \\
& \quad \leq \frac{1}{2}\left(\left\|t_{n}-p\right\|^{2}+\left\|w_{n}-p\right\|^{2}-\left\|\left(t_{n}-w_{n}\right)-\bar{\beta}\left(B t_{n}-B p\right)\right\|^{2}\right) \\
& \quad \leq \frac{1}{2}\left(\left\|t_{n}-p\right\|^{2}+\left\|w_{n}-p\right\|^{2}-\left\|t_{n}-w_{n}\right\|^{2}\right)+\frac{1}{2}\left(2 \bar{\beta}\left\langle t_{n}-w_{n}, B t_{n}-B p\right\rangle-\beta_{n}^{2}\left\|B t_{n}-B p\right\|^{2}\right),
\end{aligned}
$$

which implies that

$$
\left\|w_{n}-p\right\|^{2} \leq\left\|t_{n}-p\right\|^{2}-\left\|t_{n}-w_{n}\right\|^{2}+2 \bar{\beta}\left\langle t_{n}-w_{n}, B t_{n}-B p\right\rangle-\beta_{n}^{2}\left\|B t_{n}-B p\right\|^{2} .
$$


Journal of Applied Mathematics

Again from (3.29), we have

$$
\begin{aligned}
\left\|x_{n+1}-p\right\|^{2}= & \left\|P_{C}\left(\alpha_{n} \gamma g\left(x_{n}\right)+\left(I-\alpha_{n} A\right) S w_{n}\right)-p\right\|^{2} \\
\leq & \left\|\alpha_{n} \gamma g\left(x_{n}\right)+\left(I-\alpha_{n} A\right) S w_{n}-p\right\|^{2} \\
\leq & \left(\alpha_{n}\left\|\gamma g\left(x_{n}\right)-A p\right\|+\left(1-\alpha_{n} \bar{\gamma}\right)\left\|S w_{n}-p\right\|\right)^{2} \\
\leq & \alpha_{n}\left\|\gamma g\left(x_{n}\right)-A p\right\|^{2}+\left(1-\alpha_{n} \bar{\gamma}\right)\left\|w_{n}-p\right\|^{2} \\
& +2 \alpha_{n}\left(1-\alpha_{n} \bar{\gamma}\right)\left\|\gamma g\left(x_{n}\right)-A p\right\|\left\|w_{n}-p\right\| \\
\leq & \alpha_{n}\left\|\gamma g\left(x_{n}\right)-A p\right\|^{2}+\left(1-\alpha_{n} \bar{\gamma}\right)\left\|t_{n}-p\right\|^{2}-\left(1-\alpha_{n} \bar{\gamma}\right)\left\|t_{n}-w_{n}\right\|^{2} \\
& +2 \bar{\beta}\left(1-\alpha_{n} \bar{\gamma}\right)\left\langle t_{n}-w_{n}, B t_{n}-B p\right\rangle-\beta_{n}^{2}\left(1-\alpha_{n} \bar{\gamma}\right)\left\|B t_{n}-B p\right\|^{2} \\
& +2 \alpha_{n}\left(1-\alpha_{n} \bar{\gamma}\right)\left\|\gamma g\left(x_{n}\right)-A p\right\|\left\|w_{n}-p\right\| \\
\leq & \alpha_{n}\left\|\gamma g\left(x_{n}\right)-A p\right\|^{2}+\left\|x_{n}-p\right\|^{2}-\left(1-\alpha_{n} \bar{\gamma}\right)\left\|t_{n}-w_{n}\right\|^{2} \\
& +2 \bar{\beta}\left(1-\alpha_{n} \bar{\gamma}\right)\left\langle t_{n}-w_{n}, B t_{n}-B p\right\rangle-\beta_{n}^{2}\left(1-\alpha_{n} \bar{\gamma}\right)\left\|B t_{n}-B p\right\|^{2} \\
& +2 \alpha_{n}\left(1-\alpha_{n} \bar{\gamma}\right)\left\|\gamma g\left(x_{n}\right)-A p\right\|\left\|w_{n}-p\right\| .
\end{aligned}
$$

This implies that

$$
\begin{aligned}
\left(1-\alpha_{n} \bar{\gamma}\right)\left\|t_{n}-w_{n}\right\|^{2} \leq & \alpha_{n}\left\|\gamma g\left(x_{n}\right)-A p\right\|^{2}+\left\|x_{n}-p\right\|^{2}-\left\|x_{n+1}-p\right\|^{2} \\
& +2 \bar{\beta}\left(1-\alpha_{n} \bar{\gamma}\right)\left\langle t_{n}-w_{n}, B t_{n}-B p\right\rangle-\beta_{n}^{2}\left(1-\alpha_{n} \bar{\gamma}\right)\left\|B t_{n}-B p\right\|^{2} \\
& +2 \alpha_{n}\left(1-\alpha_{n} \bar{\gamma}\right)\left\|\gamma g\left(x_{n}\right)-A p\right\|\left\|w_{n}-p\right\| .
\end{aligned}
$$

Since $\alpha_{n} \rightarrow 0$ and $\left\|B t_{n}-B p\right\| \rightarrow 0$, we obtain that

$$
\lim _{n \rightarrow \infty}\left\|t_{n}-w_{n}\right\|=0
$$

From (1.16), we have

$$
\begin{aligned}
\left\|x_{n+1}-S w_{n}\right\| & =\left\|P_{C}\left(\alpha_{n} \gamma g\left(x_{n}\right)+\left(I-\alpha_{n} A\right) S w_{n}\right)-P_{C}\left(S w_{n}\right)\right\| \\
& \leq\left\|\alpha_{n} \gamma g\left(x_{n}\right)+\left(I-\alpha_{n} A\right) S w_{n}-S w_{n}\right\| \\
& =\alpha_{n}\left\|\gamma g\left(x_{n}\right)+A S w_{n}\right\| \longrightarrow 0, \quad \text { as } n \longrightarrow \infty
\end{aligned}
$$

Since

$$
\begin{aligned}
\left\|t_{n}-S t_{n}\right\| & \leq\left\|t_{n}-x_{n}\right\|+\left\|x_{n}-x_{n+1}\right\|+\left\|x_{n+1}-S w_{n}\right\|+\left\|S w_{n}-S t_{n}\right\| \\
& \leq\left\|t_{n}-x_{n}\right\|+\left\|x_{n}-x_{n+1}\right\|+\left\|x_{n+1}-S w_{n}\right\|+\left\|w_{n}-t_{n}\right\|
\end{aligned}
$$


from (3.7), (3.24), (3.32), and (3.33), we obtain that $\left\|t_{n}-S t_{n}\right\| \rightarrow 0$, as $n \rightarrow \infty$. Moreover, we get that

$$
\begin{aligned}
\left\|w_{n}-S w_{n}\right\| & \leq\left\|w_{n}-t_{n}\right\|+\left\|t_{n}-S t_{n}\right\|+\left\|S t_{n}-S w_{n}\right\| \\
& \leq 2\left\|w_{n}-t_{n}\right\|+\left\|t_{n}-S t_{n}\right\| \longrightarrow 0, \quad \text { as } n \longrightarrow \infty
\end{aligned}
$$

Since

$$
\left\|x_{n}-w_{n}\right\| \leq\left\|x_{n}-t_{n}\right\|+\left\|t_{n}-w_{n}\right\|
$$

it implies that

$$
\lim _{n \rightarrow \infty}\left\|x_{n}-w_{n}\right\|=0
$$

Since

$$
\begin{aligned}
\left\|x_{n}-S x_{n}\right\| & \leq\left\|x_{n}-x_{n+1}\right\|+\left\|x_{n+1}-S w_{n}\right\|+\left\|S w_{n}-S x_{n}\right\| \\
& \leq\left\|x_{n}-x_{n+1}\right\|+\left\|x_{n+1}-S w_{n}\right\|+\left\|w_{n}-x_{n}\right\|,
\end{aligned}
$$

we obtain that

$$
\lim _{n \rightarrow \infty}\left\|x_{n}-S x_{n}\right\|=0
$$

Step 6. We show that

$$
\limsup _{n \rightarrow \infty}\left\langle(r g-A) q, S w_{n}-q\right\rangle \leq 0
$$

Indeed, we choose a subsequence $\left\{w_{n_{k}}\right\}$ of $\left\{w_{n}\right\}$ such that

$$
\limsup _{n \rightarrow \infty}\left\langle(r g-A) q, S w_{n}-q\right\rangle=\lim _{k \rightarrow \infty}\left\langle(r g-A) q, S w_{n_{k}}-q\right\rangle
$$

Since $\left\{w_{n}\right\}$ is bounded, there exists a subsequence $\left\{w_{n_{k_{i}}}\right\}$ of $\left\{w_{n_{k}}\right\}$ which converges weakly to $p$. Next we show that $p \in \Omega$.

We prove that $p \in F(S)$. We may assume without loss of generality that $w_{n_{k}} \rightarrow p$. Since $\left\|w_{n}-S w_{n}\right\| \rightarrow 0$, we obtain $S w_{n_{k}} \rightarrow p$. Since $\left\|x_{n}-S x_{n}\right\| \rightarrow 0,\left\|x_{n}-w_{n}\right\| \rightarrow 0$ and by Lemma 2.5, we have $p \in F(S)$.

We show that $p \in \operatorname{EP}(f)$. From Steps 4 and 6, we have that

$$
t_{n_{k}} \rightarrow p, \quad x_{n_{k}} \rightarrow p, \quad y_{n_{k}} \rightarrow p
$$


Since $y_{n}$ is the unique solution of the convex minimization problem

$$
\min \left\{\frac{1}{2}\left\|y-x_{n}\right\|^{2}+f\left(x_{n}, y\right): y \in C\right\}
$$

we have

$$
0 \in \partial_{2}\left(\lambda_{n} f\left(x_{n}, y\right)+\frac{1}{2}\left\|y-x_{n}\right\|^{2}\right)\left(y_{n}\right)+N_{C}\left(y_{n}\right)
$$

It follows that

$$
0=\lambda_{n} z+y_{n}-x_{n}+z_{n}
$$

where $z \in \partial_{2} f\left(x_{n}, y_{n}\right)$ and $z_{n} \in N_{C}\left(y_{n}\right)$. By the definition of the normal cone $N_{C}$, we get that

$$
\left\langle y_{n}-x_{n}, y-y_{n}\right\rangle \geq \lambda_{n}\left\langle z, y_{n}-y\right\rangle, \quad \forall y \in C
$$

On the other hand, since $f\left(x_{n}, \cdot\right)$ is subdifferentiable on $C$ and $z \in \partial_{2} f\left(x_{n}, y_{n}\right)$, we have

$$
f\left(x_{n}, y\right)-f\left(x_{n}, y_{n}\right) \geq\left\langle z, y-y_{n}\right\rangle, \quad \forall y \in C \text {. }
$$

Combining (3.47) with (3.46), we have

$$
\lambda_{n}\left(f\left(x_{n}, y\right)-f\left(x_{n}, y_{n}\right)\right) \geq\left\langle y_{n}-x_{n}, y_{n}-y\right\rangle, \quad \forall y \in C
$$

Hence

$$
\lambda_{n_{k}}\left(f\left(x_{n_{k}}, y\right)-f\left(x_{n_{k}}, y_{n_{k}}\right)\right) \geq\left\langle y_{n_{k}}-x_{n_{k}}, y_{n_{k}}-y\right\rangle, \quad \forall y \in C
$$

Thus, using $\{\lambda \subset[a, b] \subset(0,1 / L)\}$ and the upper semicontinuity of $f$, we have

$$
f(p, y) \geq 0, \quad \forall y \in C
$$

Hence $p \in \operatorname{EP}(f)$.

We show that $p \in \operatorname{VI}(C, B)$. Let

$$
T v= \begin{cases}B v+N_{C} v, & v \in C, \\ \emptyset, & v \notin C,\end{cases}
$$


where $N_{C} v$ is normal cone to $C$ at $v \in C$. Then $T$ is a maximal monotone operator. Let $(v, u) \in$ $G(T)$. Since $u-B v \in N_{C} v$ and $w_{n} \in C$, we have $\left\langle v-y_{n}, u-B v\right\rangle \geq 0$. On the other hand, by Lemma 2.1(iv) and from $w_{n}=P_{C}\left(t_{n}-\bar{\beta} B t_{n}\right)$, we have

$$
\left\langle v-w_{n}, w_{n}-\left(t_{n}-\bar{\beta} B t_{n}\right)\right\rangle \geq 0,
$$

and hence $\left\langle v-w_{n},\left(w_{n}-t_{n}\right) / \bar{\beta}+B t_{n}\right\rangle \geq 0$. Therefore, we get that

$$
\begin{aligned}
\left\langle v-w_{n_{k}}, u\right\rangle & \geq\left\langle v-w_{n_{k}}, B v\right\rangle \\
& \geq\left\langle v-w_{n_{k}}, B v\right\rangle-\left\langle v-w_{n_{k}}, \frac{w_{n_{k}}-t_{n_{k}}}{\lambda_{n_{k}}}+B x_{n_{k}}\right\rangle \\
& =\left\langle v-w_{n_{k}}, B v-B t_{n_{k}}-\frac{w_{n_{k}}-t_{n_{k}}}{\lambda_{n_{k}}}\right\rangle \\
& =\left\langle v-w_{n_{k}}, B v-B w_{n_{k}}\right\rangle+\left\langle v-w_{n_{k}}, B w_{n_{k}}-B t_{n_{k}}\right\rangle-\left\langle v-w_{n_{k}}, \frac{w_{n_{k}}-t_{n_{k}}}{\lambda_{n_{k}}}\right\rangle \\
& \geq\left\langle v-w_{n_{k}}, B w_{n_{k}}-B t_{n_{k}}\right\rangle-\left\langle v-w_{n_{k}}, \frac{w_{n_{k}}-t_{n_{k}}}{\lambda_{n_{k}}}\right\rangle .
\end{aligned}
$$

This implies that $\langle v-p, u\rangle \geq 0$ as $k \rightarrow \infty$. Since $T$ is maximal monotone, we have $p \in T^{-1} 0$ and hence $p \in \operatorname{VI}(C, B)$.

From (a), (b), and (c), we obtain that $p \in \Omega$. This implies that

$$
\begin{aligned}
\limsup _{n \rightarrow \infty}\left\langle(r g-A) q, S w_{n}-q\right\rangle & =\lim _{k \rightarrow \infty}\left\langle(\gamma g-A) q, S w_{n_{k}}-q\right\rangle \\
& =\langle(\gamma g-A) q, p-q\rangle \leq 0 .
\end{aligned}
$$

Step 7. We show that $x_{n} \rightarrow q$. We observe that

$$
\begin{aligned}
\left\|x_{n+1}-q\right\|^{2}= & \left\|P_{C}\left(\alpha_{n} \gamma g\left(x_{n}\right)+\left(I-\alpha_{n} A\right) S w_{n}\right)-P_{C}(q)\right\|^{2} \\
\leq & \left\|\alpha_{n} \gamma g\left(x_{n}\right)+\left(I-\alpha_{n} A\right) S w_{n}-q\right\|^{2} \\
\leq & \alpha_{n}^{2}\left\|\gamma g\left(x_{n}\right)-A q\right\|+\left\|\left(I-\alpha_{n} A\right)\left(S w_{n}-q\right)\right\|^{2} \\
& +2 \alpha_{n}\left\langle\left(I-\alpha_{n} A\right)\left(S w_{n}-q\right), r g\left(x_{n}\right)-A q\right\rangle \\
\leq & \alpha_{n}^{2}\left\|\gamma g\left(x_{n}\right)-A q\right\|+\left(1-\alpha_{n} \bar{\gamma}\right)^{2}\left\|S w_{n}-q\right\|^{2} \\
& +2 \alpha_{n}\left\langle S w_{n}-q, r g\left(x_{n}\right)-A q\right\rangle-2 \alpha_{n}^{2}\left\langle A\left(S w_{n}-q\right), \gamma g\left(x_{n}\right)-A q\right\rangle \\
\leq & \left(1-\alpha_{n} \bar{\gamma}\right)^{2}\left\|w_{n}-q\right\|^{2}+\alpha_{n}^{2}\left\|\gamma g\left(x_{n}\right)-A q\right\| \\
& +2 \alpha_{n}\left\langle S w_{n}-q, \gamma g\left(x_{n}\right)-\gamma g(q)\right\rangle+2 \alpha_{n}\left\langle S w_{n}-q, \gamma g(q)-A q\right\rangle \\
& -2 \alpha_{n}^{2}\left\langle A\left(S w_{n}-q\right), r g\left(x_{n}\right)-A q\right\rangle
\end{aligned}
$$


Journal of Applied Mathematics

$$
\begin{aligned}
\leq & \left(1-\alpha_{n} \bar{\gamma}\right)^{2}\left\|t_{n}-q\right\|^{2}+\alpha_{n}^{2}\left\|\gamma g\left(x_{n}\right)-A q\right\| \\
& +2 \alpha_{n}\left\|S w_{n}-q\right\|\left\|\gamma g\left(x_{n}\right)-\gamma g(q)\right\|+2 \alpha_{n}\left\langle S w_{n}-q, \gamma g(q)-A q\right\rangle \\
& -2 \alpha_{n}^{2}\left\langle A\left(S w_{n}-q\right), \gamma g\left(x_{n}\right)-A q\right\rangle \\
\leq & \left(1-\alpha_{n} \bar{\gamma}\right)^{2}\left\|x_{n}-q\right\|^{2}+\alpha_{n}^{2}\left\|\gamma g\left(x_{n}\right)-A q\right\| \\
& +2 \gamma \alpha \alpha_{n}\left\|w_{n}-q\right\|\left\|x_{n}-q\right\|+2 \alpha_{n}\left\langle S w_{n}-q, \gamma g(q)-A q\right\rangle \\
& -2 \alpha_{n}^{2}\left\langle A\left(S w_{n}-q\right), \gamma g\left(x_{n}\right)-A q\right\rangle \\
\leq & \left(1-\alpha_{n} \bar{\gamma}\right)^{2}\left\|x_{n}-q\right\|^{2}+\alpha_{n}^{2}\left\|\gamma g\left(x_{n}\right)-A q\right\|^{2} \\
& +2 \gamma \alpha \alpha_{n}\left\|x_{n}-q\right\|^{2}+2 \alpha_{n}\left\langle S w_{n}-q, \gamma g(q)-A q\right\rangle \\
& -2 \alpha_{n}^{2}\left\langle A\left(S w_{n}-q\right), \gamma g\left(x_{n}\right)-A q\right\rangle \\
\leq & \left(\left(1-\alpha_{n} \bar{\gamma}\right)^{2}+2 \gamma \alpha \alpha_{n}\right)\left\|x_{n}-q\right\|^{2}+\alpha_{n}^{2}\left\|\gamma g\left(x_{n}\right)-A q\right\|^{2} \\
& +2 \alpha_{n}\left\langle S w_{n}-q, \gamma g(q)-A q\right\rangle+\alpha_{n}^{2} \bar{\gamma}^{2}\left\|x_{n}-q\right\|^{2} \\
& +2 \alpha_{n}^{2}\left\|A\left(S w_{n}-q\right)\right\|\left\|A q-\gamma g\left(x_{n}\right)\right\| \\
= & \left(1-2 \alpha_{n}(\bar{\gamma}-\gamma \alpha)\right)\left\|x_{n}-q\right\|^{2} \\
& +\alpha_{n}\left(2\left\langle S w_{n}-q, \gamma g(q)-A q\right\rangle+\alpha_{n}\left\|\gamma g\left(x_{n}\right)-A q\right\|^{2}\right. \\
& \left.+2 \alpha_{n}\left\|A\left(S w_{n}-q\right)\right\|\left\|A q-\gamma g\left(x_{n}\right)\right\|+\alpha_{n} \bar{\gamma}^{2}\left\|x_{n}-q\right\|^{2}\right) .
\end{aligned}
$$

Since $\left\{x_{n}\right\},\left\{g\left(x_{n}\right)\right\}$, and $\left\|S w_{n}\right\|$ are bounded, we can take a constant $M>0$ such that

$$
M \geq \sup _{n \geq 0}\left\{\alpha_{n}\left\|\gamma g\left(x_{n}\right)-A q\right\|^{2}+2 \alpha_{n}\left\|A\left(S w_{n}-q\right)\right\|\left\|A q-r g\left(x_{n}\right)\right\|+\alpha_{n} \bar{\gamma}^{2}\left\|x_{n}-q\right\|^{2}\right\} .
$$

This implies that

$$
\left\|x_{n+1}-q\right\|^{2} \leq\left(1-2(\bar{\gamma}-\gamma \alpha) \alpha_{n}\right)\left\|x_{n}-q\right\|^{2}+\alpha_{n} \sigma_{n}
$$

where $\sigma_{n}=2\left\langle S w_{n}-q, \gamma g(q)-A q\right\rangle+M \alpha_{n}$. From (3.40), we have lim sup $\sin _{n \rightarrow \infty} \sigma_{n} \leq 0$. Applying Lemma 2.7 to (3.57), we obtain that $x_{n} \rightarrow q$ as $n \rightarrow \infty$. This completes the proof. corollary.

If we put $\gamma=1$ and $A=I$ in Theorem 3.1, we immediately obtain the following

Corollary 3.2. Let $H$ be a real Hilbert space, and let $C$ be a closed convex subset of $H$. Let $f: C \times C \rightarrow$ $\mathbb{R}$ be a bifunction satisfying (A1)-(A5), let $B: C \rightarrow H$ be a $\beta$-inverse strongly monotone mapping, and let $g: C \rightarrow C$ be a contraction with coefficient $\alpha(\alpha \in(0,1))$. Assume that $0<\gamma<\bar{\gamma} / \alpha$. Let 
$S$ be a nonexpansive mapping of $C$ into itself such that $\Omega:=F(S) \cap \operatorname{EP}(f) \cap \operatorname{VI}(C, B) \neq \emptyset$. Let the sequences $\left\{x_{n}\right\},\left\{y_{n}\right\}$, and $\left\{t_{n}\right\}$ be generated by

$$
\begin{gathered}
x_{0}=x \in C, \\
y_{n}=\operatorname{argmin}\left\{\lambda_{n} f\left(x_{n}, y\right)+\frac{1}{2}\left\|y-x_{n}\right\|^{2}: y \in C\right\}, \\
t_{n}=\operatorname{argmin}\left\{\lambda_{n} f\left(y_{n}, y\right)+\frac{1}{2}\left\|y-x_{n}\right\|^{2}: y \in C\right\}, \\
x_{n+1}=\alpha_{n} g\left(x_{n}\right)+\left(1-\alpha_{n}\right) S P_{C}\left(t_{n}-\bar{\beta} B t_{n}\right), \quad n \geq 0,
\end{gathered}
$$

where $\left\{\alpha_{n}\right\} \subset(0,1),\{\bar{\beta}\} \subset[a, b]$ for some $a, b \in(0,2 \beta)$, and $\left\{\lambda_{n}\right\} \subset[c, d]$ for some $c, d \in(0,1 / L)$, where $L=\max \left\{2 c_{1}, 2 c_{2}\right\}$. Suppose that the following conditions are satisfied:

(B1) $\lim _{n \rightarrow \infty} \alpha_{n}=0$;

(B2) $\sum_{n=1}^{\infty} \alpha_{n}=\infty$;

(B3) $\sum_{n=1}^{\infty}\left|\alpha_{n+1}-\alpha_{n}\right|<\infty$;

(B4) $\sum_{n=1}^{\infty} \sqrt{\left|\lambda_{n+1}-\lambda_{n}\right|}<\infty$.

Then the following holds.

(i) $P_{\Omega} g$ is a contraction on $C$; and hence there exists $q \in C$ such that $q=P_{\Omega} g(q)$, where $P_{\Omega}$ is the metric projection of $H$ onto $C$.

(ii) The sequences $\left\{x_{n}\right\},\left\{y_{n}\right\}$, and $\left\{t_{n}\right\}$ converge strongly to the same point $q$ which is the unique solution in the $\Omega$ to the following variational inequality:

$$
\langle(I-f) q, x-q\rangle \geq 0, \quad \forall x \in \Omega
$$

If we put $g \equiv u$ in the previous corollary, we get the following corollary.

Corollary 3.3. Let $H$ be a real Hilbert space, and let $C$ be a closed convex subset of $H$. Let $f$ : $C \times C \rightarrow \mathbb{R}$ be a bifunction satisfying (A1)-(A5), and let $B: C \rightarrow H$ be a $\beta$-inverse strongly monotone mapping. Assume that $0<\gamma<\bar{\gamma} / \alpha$. Let $S$ be a nonexpansive mapping of $C$ into itself such that $\Omega:=F(S) \cap \operatorname{EP}(f) \cap \operatorname{VI}(C, B) \neq \emptyset$. Let the sequences $\left\{x_{n}\right\},\left\{y_{n}\right\}$, and $\left\{t_{n}\right\}$ be generated by

$$
\begin{gathered}
x_{0}=x \in C, \\
y_{n}=\operatorname{argmin}\left\{\lambda_{n} f\left(x_{n}, y\right)+\frac{1}{2}\left\|y-x_{n}\right\|^{2}: y \in C\right\}, \\
t_{n}=\operatorname{argmin}\left\{\lambda_{n} f\left(y_{n}, y\right)+\frac{1}{2}\left\|y-x_{n}\right\|^{2}: y \in C\right\}, \\
x_{n+1}=\alpha_{n} u+\left(1-\alpha_{n}\right) S P_{C}\left(t_{n}-\bar{\beta} B t_{n}\right), \quad n \geq 0,
\end{gathered}
$$


where $\left\{\alpha_{n}\right\} \subset(0,1),\{\bar{\beta}\} \subset[a, b]$ for some $a, b \in(0,2 \beta)$, and $\left\{\lambda_{n}\right\} \subset[c, d]$ for some $c, d \in(0,1 / L)$, where $L=\max \left\{2 c_{1}, 2 c_{2}\right\}$. Suppose that the following conditions are satisfied:

(B1) $\lim _{n \rightarrow \infty} \alpha_{n}=0$;

(B2) $\sum_{n=1}^{\infty} \alpha_{n}=\infty$;

(B3) $\sum_{n=1}^{\infty}\left|\alpha_{n+1}-\alpha_{n}\right|<\infty$;

(B4) $\sum_{n=1}^{\infty} \sqrt{\left|\lambda_{n+1}-\lambda_{n}\right|}<\infty$.

Then the sequences $\left\{x_{n}\right\},\left\{y_{n}\right\}$, and $\left\{t_{n}\right\}$ converge strongly to the same point $q$, where $q=P_{\Omega} u$, which is the unique solution in the $\Omega$ to the following variational inequality:

$$
\langle q-u, x-q\rangle \geq 0, \quad \forall x \in \Omega .
$$

\section{Deduced Theorems}

Let $C$ be a nonempty closed convex subset of a real Hilbert space $H$ with inner product $\langle\cdot, \cdot\rangle$. Let $F$ be a nonlinear mapping from $C$ into $H$. Recall that the function $F$ is called

(a) strongly monotone on $C$ if there exists $\beta>0$ such that

$$
\langle F(x)-F(y), x-y\rangle \geq \beta\|x-y\|^{2}, \quad \forall x, y \in C ;
$$

(b) monotone on $C$ if

$$
\langle F(x)-F(y), x-y\rangle \geq 0, \quad \forall x, y \in C ;
$$

(c) pseudomonotone on $C$ if

$$
\langle F(y), x-y\rangle \geq 0 \Longrightarrow\langle F(x), x-y\rangle \leq 0, \quad \forall x, y \in C
$$

Remark 4.1. Notice that if $F$ is $L$-Lipschitz on $C$, then for each $x, y \in C, f(x, y)=\langle F(x), y-x\rangle$ is Lipschitz-type continuous with constants $c_{1}=c_{2}=L / 2$ on $C$. Indeed,

$$
\begin{aligned}
f(x, y)+f(y, z)-f(x, z) & =\langle F(x), y-x\rangle+\langle F(y), z-y\rangle-\langle F(x), z-x\rangle \\
& =-\langle F(y)-F(x), y-z\rangle \\
& \geq-\|F(y)-F(x)\|\|y-z\| \\
& \geq-L\|x-y\|\|y-z\| \\
& \geq-\frac{L}{2}\|x-y\|^{2}-\frac{L}{2}\|y-z\|^{2} \\
& =-c_{1}\|x-y\|^{2}-c_{2}\|y-z\|^{2} .
\end{aligned}
$$

Thus $f$ is Lipschitz-type continuous on $C$. 
Let $f: C \times C \rightarrow \mathbb{R}$ be defined by $f(x, y)=\langle F(x), y-x\rangle$, where $F: C \rightarrow H$. Thus, by Algorithm (1.15), we get the following:

$$
\begin{aligned}
y_{n} & =\operatorname{argmin}\left\{\lambda_{n} f\left(x_{n}, y\right)+\frac{1}{2}\left\|y-x_{n}\right\|^{2}: y \in C\right\} \\
& =\operatorname{argmin}\left\{\lambda_{n}\left\langle F\left(x_{n}\right), y-x_{n}\right\rangle+\frac{1}{2}\left\|y-x_{n}\right\|^{2}: y \in C\right\} \\
& =P_{C}\left(x_{n}-\lambda_{n} F\left(x_{n}\right)\right) .
\end{aligned}
$$

Similarly, we also obtain that $t_{n}=P_{C}\left(x_{n}-\lambda_{n} F\left(y_{n}\right)\right)$. Applying Theorem 3.1, we obtain the convergence theorem for finding a common element of the set of fixed points of a nonexpansive mapping and the solution set $\mathrm{VI}(C, B)$.

Corollary 4.2. Let $H$ be a real Hilbert space, and let $C$ be a closed convex subset of $H$. Let $F: C \rightarrow H$ be a monotone, L-Lipschitz continuous mapping, let $B: C \rightarrow H$ be a $\beta$-inverse strongly monotone mapping, also let $A$ be a strongly positive linear bounded operator of $H$ into itself with coefficient $\bar{\gamma}>0$ such that $\|A\|=1$, and let $g: C \rightarrow C$ be a contraction with coefficient $\alpha(\alpha \in(0,1))$. Assume that $0<\gamma<\bar{\gamma} / \alpha$. Let $S$ be a nonexpansive mapping of $C$ into itself such that $\Omega=F(S) \cap \operatorname{EP}(f) \cap$ $\mathrm{VI}(C, B) \neq \emptyset$. Let the sequence $\left\{x_{n}\right\},\left\{y_{n}\right\}$, and $\left\{t_{n}\right\}$ be generated by

$$
\begin{gathered}
x_{0}=x \in C, \\
y_{n}=P_{C}\left(x_{n}-\lambda_{n} F\left(x_{n}\right)\right), \\
t_{n}=P_{C}\left(x_{n}-\lambda_{n} F\left(y_{n}\right)\right), \\
x_{n+1}=P_{C}\left(\alpha_{n} \gamma g\left(x_{n}\right)+\left(I-\alpha_{n} A\right) S P_{C}\left(t_{n}-\bar{\beta} B t_{n}\right)\right), \quad n \geq 0,
\end{gathered}
$$

where $\left\{\alpha_{n}\right\} \subset(0,1),\{\bar{\beta}\} \subset[a, b]$ for some $a, b \in(0,2 \beta)$, and $\left\{\lambda_{n}\right\} \subset[c, d]$ for some $c, d \in(0,1 / L)$. Suppose that the following conditions are satisfied:

(B1) $\lim _{n \rightarrow \infty} \alpha_{n}=0$;

(B2) $\sum_{n=1}^{\infty} \alpha_{n}=\infty$;

(B3) $\sum_{n=1}^{\infty}\left|\alpha_{n+1}-\alpha_{n}\right|<\infty$;

(B4) $\sum_{n=1}^{\infty} \sqrt{\left|\lambda_{n+1}-\lambda_{n}\right|}<\infty$.

Then the sequences $\left\{x_{n}\right\},\left\{y_{n}\right\}$, and $\left\{t_{n}\right\}$ converge strongly to the same point $q$, where $q=P_{\Omega}(I-A+$ $r g)(q)$.

\section{Coflict of Interests}

The authors declare that they have no conflict interests.

\section{Authors' Contribution}

All authors read and approved the final paper. 


\section{Acknowledgments}

The authors would like to thank the referees for reading this paper carefully, providing valuable suggestions and comments, and pointing out a major error in the original version of this paper. Finally, the first author is supported by the Centre of Excellence in Mathematics under the Commission on Higher Education, Ministry of Education, Thailand.

\section{References}

[1] F. Deutsch and I. Yamada, "Minimizing certain convex functions over the intersection of the fixed point sets of nonexpansive mappings," Numerical Functional Analysis and Optimization, vol. 19, no. 1-2, pp. 33-56, 1998.

[2] H.-K. Xu, "Iterative algorithms for nonlinear operators," Journal of the London Mathematical Society Second Series, vol. 66, no. 1, pp. 240-256, 2002.

[3] H. K. Xu, "An iterative approach to quadratic optimization," Journal of Optimization Theory and Applications, vol. 116, no. 3, pp. 659-678, 2003.

[4] I. Yamada, "The hybrid steepest descent method for the variational inequality problem of the intersection of fixed point sets of nonexpansive mappings," in Inherently Parallel Algorithm for Feasibility and Optimization, D. Butnariu, Y. Censor, and S. Reich, Eds., pp. 473-504, Elsevier, 2001.

[5] G. Marino and H.-K. Xu, "A general iterative method for nonexpansive mappings in Hilbert spaces," Journal of Mathematical Analysis and Applications, vol. 318, no. 1, pp. 43-52, 2006.

[6] A. Moudafi, "Viscosity approximation methods for fixed-points problems," Journal of Mathematical Analysis and Applications, vol. 241, no. 1, pp. 46-55, 2000.

[7] C. Klin-eam and S. Suantai, "A new approximation method for solving variational inequalities and fixed points of nonexpansive mappings," Journal of Inequalities and Applications, vol. 2009, Article ID 520301, 16 pages, 2009.

[8] S. Takahashi and W. Takahashi, "Viscosity approximation methods for equilibrium problems and fixed point problems in Hilbert spaces," Journal of Mathematical Analysis and Applications, vol. 331, no. 1, pp. 506-515, 2007.

[9] R. T. Rockafellar, "On the maximality of sums of nonlinear monotone operators," Transactions of the American Mathematical Society, vol. 149, pp. 75-88, 1970.

[10] P. Daniele, F. Giannessi, and A. Maugeri, Equilibrium Problems and Variational Models, vol. 68 of Nonconvex Optimization and Its Applications, Kluwer Academic Publishers, Norwell, Mass, USA, 2003.

[11] P. N. Ahn, "A hybrid extragradient method extended to fixed point problems and equilibrium problems," Optimization, vol. 2011, pp. 1-13, 2011.

[12] K. Goebel and W. A. Kirk, Topics in Metric Fixed Point Theory, vol. 28 of Cambridge Studies in Advanced Mathematics, Cambridge University Press, Cambridge, Mass, USA, 1990.

[13] H.-K. Xu, "Viscosity approximation methods for nonexpansive mappings," Journal of Mathematical Analysis and Applications, vol. 298, no. 1, pp. 279-291, 2004. 


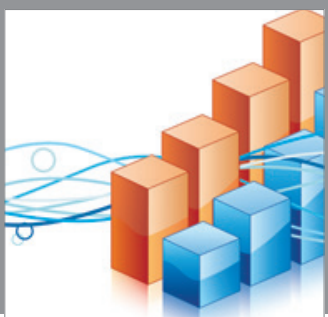

Advances in

Operations Research

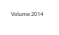

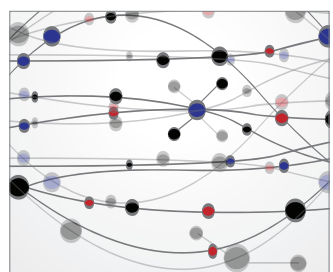

\section{The Scientific} World Journal
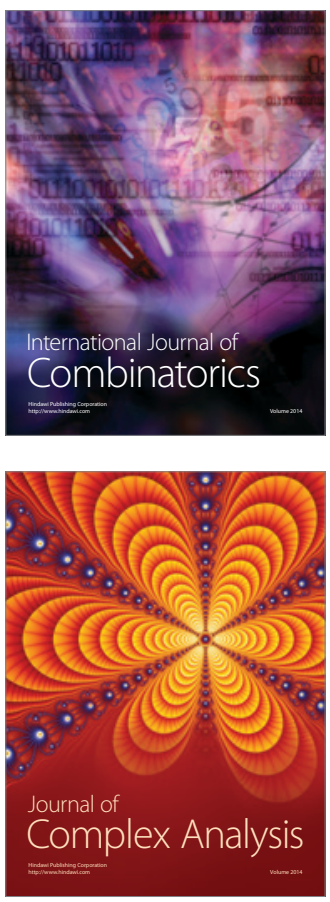

International Journal of

Mathematics and

Mathematical

Sciences
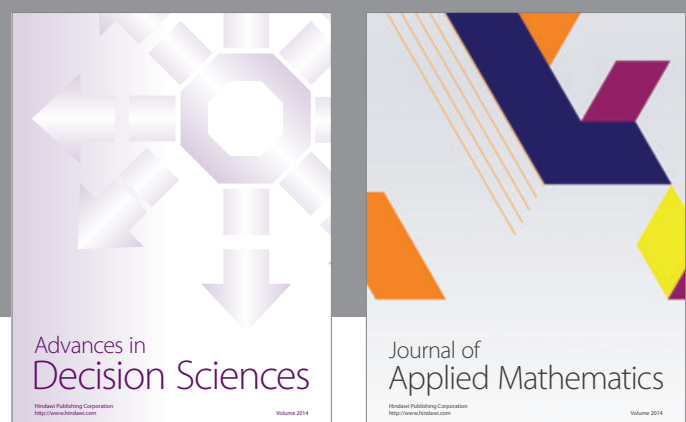

Journal of

Applied Mathematics
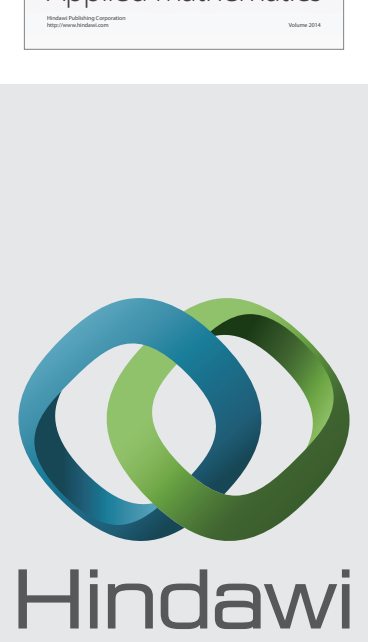

Submit your manuscripts at http://www.hindawi.com
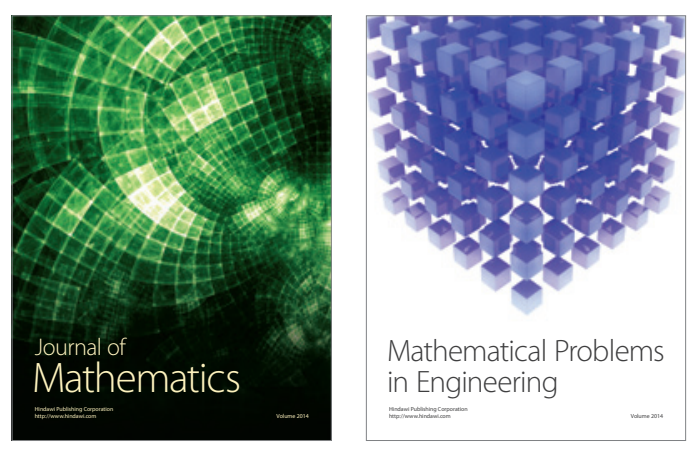

Mathematical Problems in Engineering
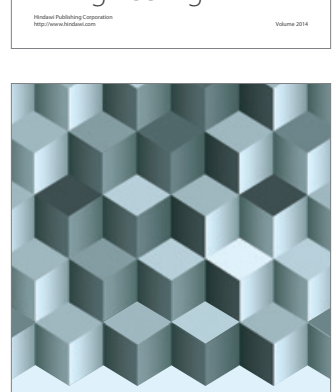

Journal of

Function Spaces
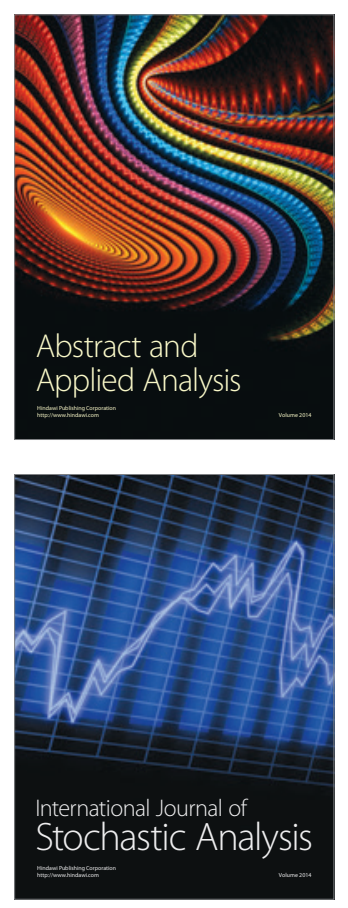

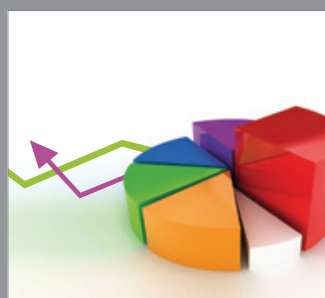

ournal of

Probability and Statistics

Promensencen
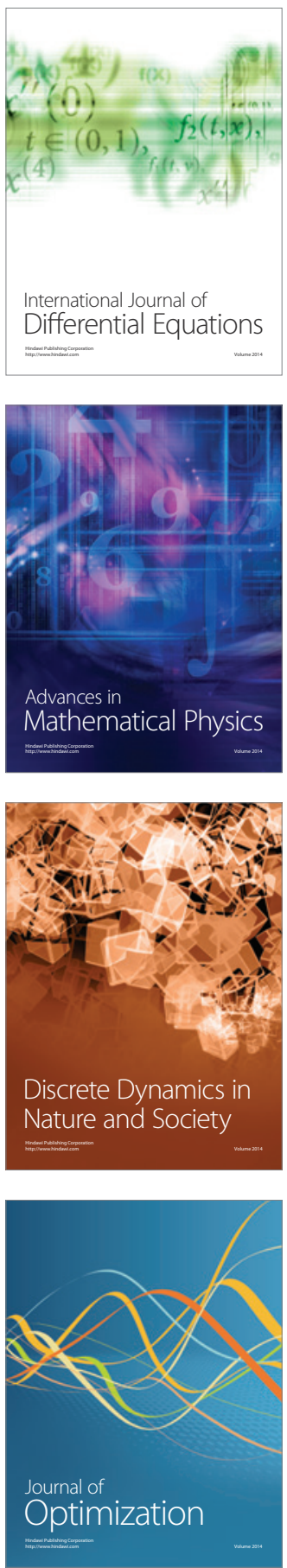